\title{
On the brink of jamming: Granular convection in densely filled containers
}

\author{
Frank Rietz and Ralf Stannarius \\ Otto-von-Guericke-University, D-39106 Magdeburg, Germany
}

(Dated: June 16, 2017)

\begin{abstract}
Granulates are ubiquitous in nature and technology, but despite their great importance, their dynamics are by far less well understood than those of liquids. We demonstrate in an almost compactly filled flat (Hele-Shaw) cell, where slow horizontal rotation simulates a variable gravitational force, that unexpected dynamic structures may arise under geometrical restrictions. The cell motion drives regular flow in the compact interior, and convection rolls combine with segregation. The container fill level is crucial for the dynamic regime. A transition from chute flow at lower fill levels to convection in densely packed containers is found. These observations suggest the existence of comparable phenomena in situations where so far no systematic search for dynamic patterns has been performed.

PACS numbers: $45.70 . \mathrm{Mg}, 45.70 . \mathrm{Qj}, 05.65 .+\mathrm{b}$,
\end{abstract}

The broad interest in the fundamental physics of granulates is motivated by their great relevance in everyday life, in particular in the processing and handling of food, pharmaceuticals and chemicals [1]. The complexity of granulates has so far withstood a description within a unified theory. Phenomena related to dense flow and jamming have become central topics of research activities [2, 3]. During the past decade, there has also been an increased interest in pattern formation of granulates [4, 5]. One of the unique, counterintuitive features of granular mixtures, for example, is their segregation under external excitation. Among the classical systems are e.g. the horizontally rotating mixer $[\underline{6}$ ] and horizontally or vertically vibrated layers [7-12]. In most experiments, the rearrangement of individual grains in the container requires the fluidization of the granular bed, but slow fluxes can occur even in deep layers where the grains do not have individual degrees of freedom for positional changes. An impressive example is patterning of permafrost ground by freeze-thaw cycles [13].

In densely packed systems, geometrical restrictions prevent the independent motion of particles, and slow and collective dynamics are observed, as in the dynamic glass transition of supercooled liquids [3]. Very few studies consider such systems as e.g. densely filled vibrating containers [9] or horizontally rotating cylindrical mixers [14, 15]. Access to the inner structure of the granular bed is often limited to invasive methods, and conclusions on subsurface convection have to be derived indirectly. A direct hint to the bulk dynamics of such systems comes from numerical studies. Simulations of disks in a flat, horizontally rotating cell [16] revealed a global convection flow. This motivated our experimental study of a flat, almost completely filled cell rotating slowly about a horizontal axis. We observe unexpected regular convection structures in combination with size segregation of the granulate (see Fig. 1 and Ref. [17]).

The measurements are performed in a transparent Hele-Shaw cell (Fig. 1, top) with a thickness of $D=5 \mathrm{~mm}$, corresponding to a few particle diameters. The cell length in axial direction is $d=500 \mathrm{~mm}$, the height $h$ is variable between $40 \mathrm{~mm}$ and $110 \mathrm{~mm}$. The dry granulate consists of spherical glass beads (Würth Ballotini MGL) with density $2.46 \mathrm{~g} / \mathrm{cm}^{3}$. Bimodal mixtures contain equal weight fractions of small $(250-350 \mu \mathrm{m}$ diameter) and large (800-900 $\mu \mathrm{m}$ diameter) beads. The cell is filled with the well mixed granulate, sealed, and thereafter rotated about the long horizontal axis. The rotational speed is $20 \mathrm{rpm}$. The Froude number, representing the ratio of centrifugal and gravitational forces, is well below 0.025 , thus centrifugal forces are negligible. In the flat cell geometry, the principal effect of the rotation is a periodic modulation of the effective gravitational acceleration, in combination with friction of the granulate at the two plates of the Hele-Shaw cell. The system is in some respect similar to the vertically shaken container [7-11]. We note in passing that unlike in recently described convection rolls in a Taylor-Couette experiment [18], the granulate is not slackened by air flow here.

First, it is necessary to define a quantitative measure for the fill level. The motional degrees of freedom of the granular particles are controlled by the available space above the granulate. However, this free volume is not a practicable parameter, since the height of the granular bed depends on the filling procedure, and it is not exactly preserved during the experiment. Therefore we weigh the granulate before filling. Using the mass and known density, we define the fill ratio $C$ as the proportion of the net volume of glass beads to the cell volume. Note that, by this definition, the free volume above the granulate for a given $C$ increases with the container height $h$. For a cell completely filled with the bimodal mixture, without and with additional manual tapping, we find $C \approx 0.66$ and $C \approx 0.70$, respectively.

The cell is observed in transmission with uniform background illumination. Images are automatically recorded every 20 revolutions: rotation is paused when the cell is in vertical position and a picture is taken with a CCD camera (KODAK Megaplus 6.3i, $3072 \times 2048$ pixels). A typical experiment runs for more than 10,000 rotations (one to a few days).

The collective particle motion is exploited for the de- 

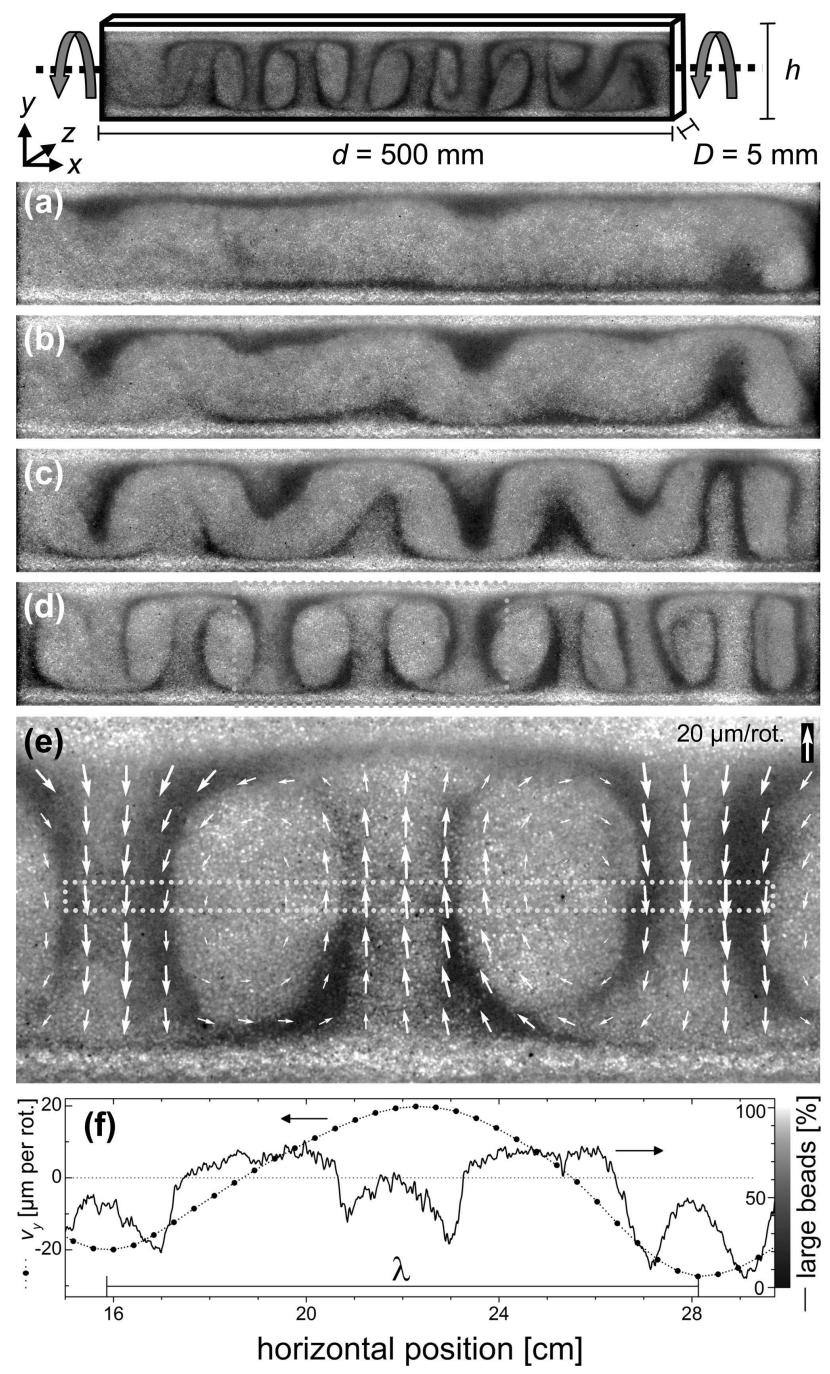

FIG. 1: Convection patterns in a densely filled cell. Top: Sketch of the experimental geometry ('front view' of the cell), the illumination source is behind the cell, the camera is positioned in front. The free volume above the granulate represents a few percent of the cell volume. (a-d) Development of the pattern after (a) 2,000, (b) 4,000, (c) 6,000 and (d) 12,000 rotations. Regions enriched with small particles appear darker, regions enriched with large particles appear brighter, the optical transmission reflects an average particle concentration through the cell depth. $C=0.647>C_{c}$, $h=80 \mathrm{~mm}$. (e) Detail of the texture of (d) and superimposed arrows of the flow field, i.e. the mean displacement of clusters per rotation, as detected from velocimetry (see text). (f) Vertical flow velocity (dots) and composition of the mixture (solid line) in the central region of (e). The local composition has been determined from the optical transmission intensity. The wavelength $\lambda$ of the flow pattern is indicated.

termination of local cluster velocities. Images are subdivided into evaluation squares of $8 \mathrm{~mm} \times 8 \mathrm{~mm}$ and, within each segment, the principal cluster displacement (maximum cross-correlation vector) is determined from the analysis of consecutive images. This method allows a reconstruction of the local granulate motion in the cell plane. Furthermore, the relation between the local transmission intensity and granular composition has been established in separate experiments.

The redistribution of particles during the cell rotation is basically restricted to the sliding of the granulate, driven by gravity, when the cell plane tilts out of the horizontal. Two qualitatively different regimes are found, depending on the fill ratio $C$. Below some critical $C_{c}$ (e.g. $C_{c} \approx 0.60$ for the $h=80 \mathrm{~mm}$ cell), chute flow occurs twice in each rotation cycle. Its result is the spontaneous formation of an axial segregation pattern (Fig. 21) of alternating stripes of small and large particles. A similar phenomenon is well known from rotating cylindrical mixers [6]. In the course of the experiment this segregation pattern slowly coarsens, finally leaving two or three well segregated regions.

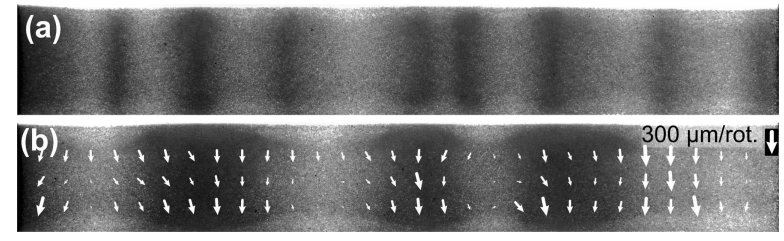

FIG. 2: Axially segregated stripe pattern in the chute flow regime, (a) after formation of the segregation stripes, 1,500 rotations, (b) coarsening after 15,000 rotations. Arrows symbolize the flow on the front side. $C=0.581<C_{c}, h=80 \mathrm{~mm}$

At $C_{c}$, the chute flow disappears and the granulate jams. At fill ratios above $C_{c}$, one observes the formation of characteristic zones after the start of the experiment [Fig. 1(a)]. In shallow layers within a few $\mathrm{mm}$ of the top and bottom cell edges, the particles' motion is fast and relatively uncorrelated. This region becomes enriched with large size beads, indicated in Fig. 1(a-d) by the bright horizontal stripes. Except for these regions, the grains can manoeuvre only in compact clusters ('glassy dynamics'). Within the next few $\mathrm{mm}$, narrow bands of jammed small size beads develop along each edge, while the central part of the cell contains mixed granulate [Fig. 1(a)]. A close inspection shows that dark regions contain almost completely segregated small beads, while the bright regions resemble a mix dominated by the large species. This initial structure becomes distorted by the onset of convective flow so that small particles from the outer bands are advected towards the center [Fig. [1(b)], and a serpentine structure develops [Fig. 1(c)]. Convection can start anywhere in the container. The emerging rolls are decorated by small particles [Fig. 1(d)]. The flow and composition patterns seen on the front extend through the whole cell depth and there are only minor differences between front and back textures. The described patterns and dynamics are qualitatively different from those at low fill ratios. The periodicity of the convection pattern, once it has formed, is persistent, and there is no coarsening. We note that different rotation speeds (be- 


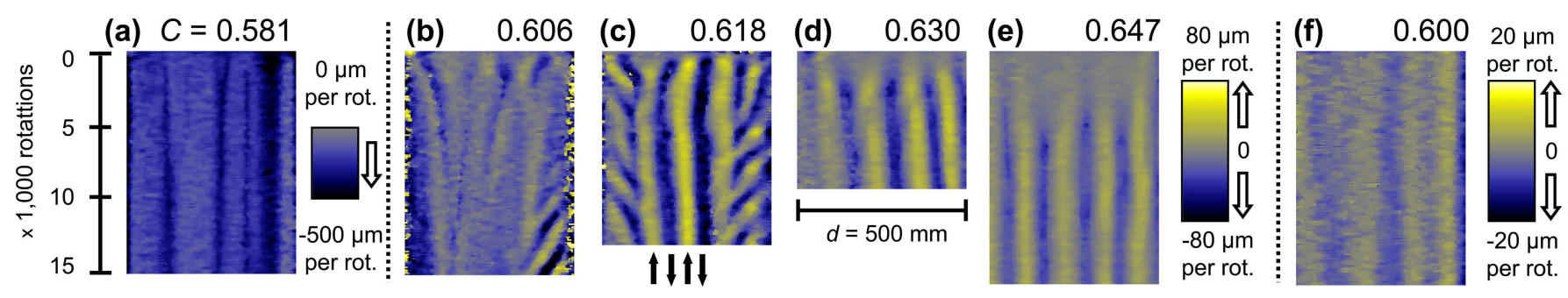

FIG. 3: (Color online) Time-space plots of the vertical flow field component $v_{y}$ in the central axis of the cell. (a-e) bidisperse mixture, $C_{c} \approx 0.60$, and (f) monodisperse granulate. Yellow (bright) indicates upflow, blue (dark) marks downflow. $(h=80 \mathrm{~mm})$ (a) global downward flow at a slightly subcritical $C$ (see Fig. 2), (b-e) convective flow in dependence on the fill ratio $C$. The flow field is uniform through the cell (in viewing direction), except for a very small global downstream component on the front side, of a few percent of the convection amplitude. When the cell is rotated by $180^{\circ}$, up and down stream regions are interchanged. Images of Fig. 1 (a-d) correspond to the space-time plot (e). (f) Flow field in a nearly monodisperse granulate. Note that monodisperse granulate has a lower packing density, the free volume above the granulate is comparable to that in (d).

tween 5 and $20 \mathrm{rpm}$ ) or evacuation of the interstitial air (between 9 and $101 \mathrm{kPa}$ ) have no influence on this general behavior. Similar convection patterns are observed for other materials too (e.g. glass bead and poppy seed mixtures, sand mixtures).

For a quantitative analysis, we focus on the velocity field, determined from the displacement of clusters as described above. Convective flow slowly sets in after the start of the experiment. Figure 1(e) shows exemplarily the flow field in a section of the cell. In the two fluidized layers at the top and bottom edges, the flow field could not be determined. The particle motion is rather uncorrelated there, and one to two orders of magnitude faster than in the clusters. Figure 1(f) shows the velocity $v_{y}$ (dots) and the local composition of the mixture (solid line) obtained from the optical transmission intensity in the middle part of Fig. 1(e), vertically averaged between the dashed lines.

The vertical velocity $v_{y}(x, h / 2)$ in the central cut is presented in Figs. 3(a-e) for different fill ratios. Figure 3(a) is a space-time plot of the flow field at subcritical $C=0.581$ for an axially segregated stripe pattern. The global downward flow reflects a small effective difference of the motion at front and rear planes, i.e. an effective vortex in the $y z$ plane, in the same sense as the cell rotation. Size segregation modulates the flow: in general the regions of higher concentrations of small particles move faster. Figures 3(b-e) evidence the qualitative change to the convection regime; the convection rolls are manifest in spatially alternating up and down flows. Slightly above $C_{c}$, there is a strong oscillation at the lateral edges of the cell [Fig. 3(c)], possibly reflecting a competition of rudimentary chute flow with the convection pattern. At higher fill ratios, these oscillations vanish. The velocity amplitude decreases with increasing $C$, and the particle motion becomes more and more collective. When $C$ approaches a value of about 0.70 , no motion is observable any more. Maximum velocities are of the order of $50 \mu \mathrm{m} /$ revolution. Thus, a cluster needs several thousand cell rotations for a full turn. There is a small constant negative offset of $v_{y}$, more than one order of magnitude smaller than the flow maxima.

From a Fourier analysis of $v_{y}(x, h / 2)$, the mode selection is monitored. Boundary conditions require that rolls end with up or down flow at the cell sides, we observe only half-integer and integer modes. A selected wave number $q_{x}=d / \lambda=4.5$ in Fig. 3(e) corresponds to 4.5 roll pairs. In most cases, the fastest growing mode dominates from the beginning and determines the wavelength of the pattern. In some experiments, the selected wavelength fluctuates between neighboring modes. The spatial period of the flow structures is not sensitive to the fill ratio above $C_{c}$. Figure 4 shows the influence of the container height on the selected wave number. The dots label runs where the selected mode was constant, while vertical bars indicate that the pattern showed temporal fluctuations of the selected mode during the experiment. As in typical hydrodynamic convection structures (e.g. Rayleigh-Bénard) the selected wavelength is roughly equal to twice the cell height. The relation $q_{x}=d /(2 h)$ that would correspond to circular convection rolls is indicated by a dashed line. The general trend is, in view of the small aspect ratio of the experiment, in reasonable agreement with such an assumption.

Finally, we have tested whether the convective flow is triggered by the segregation of the granular mixture or whether the segregation of the mixture is a consequence of the convective flow. For that purpose, a cell was filled with the nearly monodisperse beads of the large species ( $<10 \%$ radius variation). It is evident from Fig. $3(\mathrm{f})$ that a convection structure of similar wavelength as in the mixture is formed, but with considerably lower flow amplitude. Obviously the bidispersity of the mixture strongly enforces the convection pattern, and the decoration by the segregation structures facilitates the optical recognition of the pattern. However, the mechanism works in a nearly monodisperse granulate as well, though much less effectively.

The increasing collectivity of particle motion in clusters with increasing container fill ratio in the rotating 


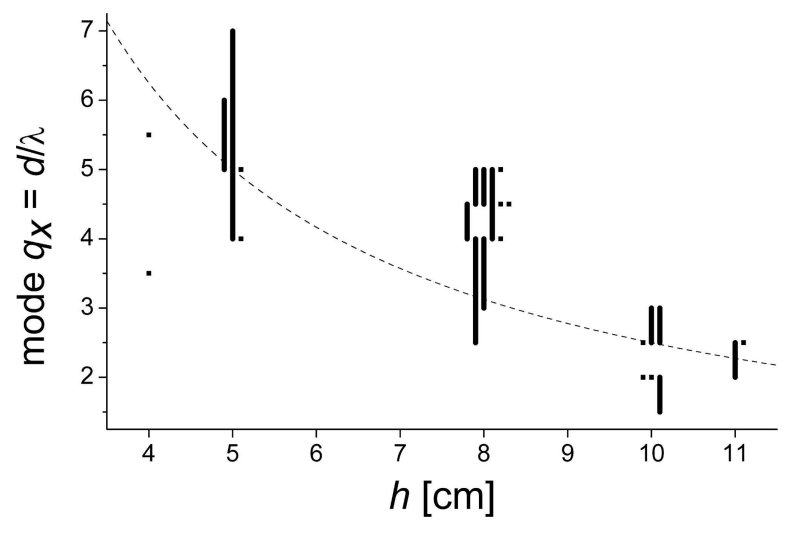

FIG. 4: Range of observed modes in dependence upon the cell height $h$. The data represent 24 individual experiments. The dashed curve symbolizes the relation for circular rolls, $q_{x}=d /(2 h)$. For clarity, individual experiments for the same cell height are slightly displaced on the abscissa.

Hele-Shaw cell coincides with observations in vibrated beds [12]. We observe the regular convection patterns in the high fill ratio range $(C$ between the upper bound chute flow limit, $\approx 0.60$, and the maximum achieved fill level, $\approx 0.70)$. This convection looks morphologically similar to familiar structures in vibrated containers [811], but irrespective of this superficial similarity there are striking differences. In the vibration experiments, up and down accelerations are asymmetric. Friction at the side walls plays an essential role, and the rolls appear gen- erally pairwise [8 11]. A critical acceleration is required [10], and the observed segregation patterns [11] are different. Similar to our experiment, convection is suppressed in the vibrated container when the free volume above the granular bed is sufficiently reduced [9].

Three mechanisms for granular convection have been discussed in literature [4, 10, 11], related to inhomogeneous agitation, friction at lateral (left/right) container ends, and interactions with the interstitial fluid, respectively. These are apparently irrelevant in our experiment. The slow dynamics of the granulate, and its practical independence of the revolution rate, suggest that dissipation does not play a significant role in the pattern formation. Rather, local fluctuations of the packing density could be relevant. Simulations [16] for a 2D cell are in a certain qualitative agreement with our experimental results, some arguments for a convection mechanism are given, but they provide no direct clue on the nature of the instability mechanism in our experiment.

Even though data for a truly three-dimensional system are not available, there are indirect indications that convection structures in compactly filled containers are found in 3D systems too [15]. The experiments presented suggest the existence of slow subsurface currents in similar situations that are not accessible to a direct optical analysis, and/or involve long, geological [19], time scales.

The authors are grateful to Tilo Finger, Nico Fricke, Jesse Gryn, Ulf Schaper, Stefan Scharfenberg, Matthias Schröter and Christian Warnke for their advice and technical assistance. This study was funded in part by a Landesstipendium Sachsen-Anhalt.
[1] T. M. Knowlton et al., Chem. Eng. Prog. 90, 44 (1994).

[2] O. Pouliquen, Phys. Rev. Lett. 93, 248001 (2004); GDR MiDi, Eur. Phys. J. E 14, 341 (2004).

[3] A. J. Liu and S. R. Nagel, Nature 396, 21 (1998); V. Trappe et al., Nature 411, 772 (2001); G. D'Anna and G. Gremaud, Nature 413, 407 (2001); P. Richard et al., Nat. Mater. 4, 121 (2005).

[4] H. M. Jaeger and S. Nagel, Rev. Mod. Phys. 68, 1259 (1996).

[5] J. Kakalios, Am. J. Phys. 73, 8 (2005); I. S. Aranson and L. S. Tsimring, Rev. Mod. Phys. 78, 641 (2006); J. M. Ottino, Chem. Eng. Sci. 61, 4165 (2006).

[6] K. M. Hill, A. Caprihan and J. Kakalios, Phys. Rev. Lett. 78, 50 (1997); D. Levine, Chaos 9, 573 (1999).

[7] A. Götzendorfer et al., Phys. Rev. E 74, 011304 (2006).

[8] C. R. Wassgren, C. E. Brennen and M. L. Hunt, Trans. ASME 63, 712 (1996); K. M. Aoki et al., Phys. Rev. E 54, 874 (1996); J. B. Knight, Phys. Rev. E 55, 6016 (1997).

[9] G. Caballero et al., in Unifying Concepts in Granular Media and Glasses, edited by A. Coniglio et al. (Elsevier, Amsterdam 2004)
[10] G. H. Ristow, Pattern Formation in Granular Materials (Springer, Berlin 2000), Chap. 3

[11] A. Kudrolli, Rep. Prog. Phys. 67, 209 (2004).

[12] P. M. Reis and T. Mullin, Phys. Rev. Lett. 89, 244301 (2002); J. Galanis et al., Phys. Rev. Lett. 96, 028002 (2006).

[13] M. A. Kessler and B. T. Werner, Science 299, 380 (2003).

[14] M. Nakagawa, J. L. Moss and S. A. Altobelli, in Physics of dry Granular Media edited by H. J. Herrmann et al. (Kluwer Acad., Dordrecht 1998); J. L. Turner and M. Nakagawa, Powder Technol. 113, 119 (2000).

[15] H. P. Kuo, P. Y. Shih and R. C. Hsu, AIChE J. 52, 2422 (2006)

[16] A. Awazu, Phys. Rev. Lett. 84, 4585 (2000).

[17] See EPAPS Document No. E-PRLTAO-100-060807 for supplementary movies. For more information on EPAPS, see http://www.aip.org/pubservs/epaps.html.

[18] S. L. Conway, T. Shinbrot, B. J. Glasser, Nature 431, 433 (2004).

[19] P. Ortoleva, Geochemical Self-Organization (Oxford Univ. Press, Oxford 1994), Chap. 14 